\title{
Building a Low-Cost Soyuz Simulator to Teach Orbital Navigation
}

\author{
Ignaty Romanov-Chernigovsky \\ International Space University \\ 1, rue J. D. Cassini, 67400, Strasbourg, France \\ iromanovchernigovsky@community.isunet.edu
}

\author{
Prof. Gongling Sun \\ International Space University \\ 1, rue J. D. Cassini, 67400, Strasbourg, France \\ gongling.sun@isunet.edu
}

\begin{abstract}
Space flight presents many dynamics unseen in any other domain. As such, they can be often hard to teach, and to have students develop an intuition for, even at a university level. A great benefit of cost efficient low-fidelity simulators is that they can enable users to directly interact with complex situations such as those encountered during space missions, whilst keeping a low learning curve for the user thanks to abstraction or simplification of the situation in question. Simulators can enable their users to start developing an intuitive understanding of complex topics such as orbital rendezvous, spacecraft docking, re-entry and navigation by instruments after only a few hours.
\end{abstract}

This paper presents the design and build process, as well as the use cases of a low-fidelity simulator for the Soyuz TMA spacecraft built at the International Space University for educational purposes. The simulator is designed to provide a simplified and cost-efficient rendition of the on-board instruments and controls in a Soyuz TMA spacecraft descent capsule using commercially available components and allow students to manually perform inorbit maneuvers that astronauts might perform over the course of a real mission. The paper focuses on design challenges associated with developing a user-friendly simulator without losing important aspects of how the Soyuz is controlled and behaves, discussing design decisions and trade-offs performed. The exact hardware and software architecture used in the final version of the simulator is also detailed.

\section{Keywords—simulator; Soyuz; education}

\section{INTRODUCTION}

Since the very first humans started training to launch into space, spacecraft simulators have been used to prepare astronauts for missions. By creating a simulacrum of a spacecraft on the ground, in a controlled environment, a powerful training tool can be created within which both nominal and dangerous scenarios can be simulated [1]. The trainees can then intuitively learn the proper actions to take in diverse situations while staying in a safe environment, rehearsing all aspects of spacecraft operation [2]. For such applications the simulators are made to be "high-fidelity", replicating accurately the parts of the spacecraft being simulated, and require the user to already hold deep knowledge of the systems being simulated.

While high-fidelity simulators are irreplaceable tools for real space training, simpler "low-fidelity" simulators that only broadly simulate real spacecraft can also be used to teach or study some aspects of spaceflight without requiring extensive training on the part of the users. The Institute of Space Systems
(IRS) at the University of Stuttgart has demonstrated this with their low-fidelity Soyuz simulator, which is used in their aerospace-engineering course, and has been used in studies about the effects of prolonged missions on spacecraft piloting skills [3] [4].

This paper presents a cheap and relatively portable simulator design (total mass under $30 \mathrm{~kg}$ ), built using about $550 €$ of components that can simulate any stage of a Soyuz mission, and provide a student or research subject with similar manual and automatic control options that the pilot would have, using a lowfidelity control interface inspired by the Soyuz TMA. An indepth report on the simulator, including all information necessary to recreate it, can be found in [5].

\section{DESIGN CHOICES}

When considering different design possibilities, the main driving factors were low learning curve, low parts cost and the immersion factor of the simulator. "Learning curve" is defined as the difficulty that a user with only basic knowledge of the Soyuz's operating principles will experience when using the simulator for the first time. How intuitive the user will find the simulator controls and interface strongly influenced the design. "Immersion" refers to how much (from the user's perspective) their experience felt similar to piloting a real spacecraft. It is an important factor in keeping the user engaged with the simulator, and increases the simulator's effect on the user's interest in spacecraft physics and engineering.

The simulator's design process was driven by the aforementioned factors, and heavily inspired by the IDS panel of Soyuz TMA and the Soyuz simulator at IRS in Stuttgart, with significant simplifications.

\section{A. Software Design Choices}

The software core of the simulator is built using the Orbiter 2010 Space Flight Simulator, developed by Martin Schweiger of UCL [6]. Orbiter presents a highly versatile framework for simulating, rendering and controlling the movements of spacecraft within a simulated solar system under the influence of realistic Newtonian Physics. While these features are crucial for the development of a simulator, what makes Orbiter particularly suited for use in a simulator setup is its highly modular software stack and the way it allows the user to interact with spacecraft instruments.

"Modules" within Orbiter allow for the creation of add-ons controlling the behaviour of different software components such 
as spacecraft, planets, instruments, etc. (cf. Fig. 2) and "plugins" are fully separate from Orbiter's core software and files, allowing for entirely new functionality unanticipated by the original developer to be seamlessly integrated into the software without conflicting with other such modifications. These features allow the community to develop countless add-ons that all work well together, making Orbiter perfect for an easy-tobuild simulator.

The simulator designed uses the Soyuz v.1.0 add-on that implements the Soyuz TMA and certain internal systems with high accuracy, such as the Kurs docking radar, functional solar arrays and batteries and individual manoeuvring thrusters [7]. A highly-detailed ISS model is provided by the ISS v3.2 add-on [8]. These add-ons were used based on the design suggestions given in [9].

The other important feature of Orbiter is the "MFD" or "Multi-Functional Display" approach for simulating onboard spacecraft instruments. The user can open different instruments in windows called "MFDs" and interact with each instrument via a set of up to 12 mouse-clickable buttons. Orbiter comes with a variety of instruments built-in that can be used to help with atmospheric flight, orbital rendezvous planning, executing accurate orbital plane changes, docking, etc. Instruments can also control spacecraft systems, allowing for high-level usercontrolled behaviour like pre-programmed manoeuvres or even full autopilot behaviour, making them very similar to the IDS in the Soyuz spacecraft.

Each Instrument has 6 input buttons on the left and right side, with 1 to 3 characters used to describe their functionality. The functions of the buttons vary dynamically with the instrument's state, depending on the instrument selected. The bottom 3 buttons are used for switching instruments, displaying options and turning the instrument panel on/off.

Orbiter also allows easy set-up of custom simulation scenarios. Any specific time (past or future) may be defined, and any number of spacecrafts placed in specific orbits or at particular places on a planet's surface.

\section{B. Hardware Choices}

In order to provide the user with a visual control layout somewhat similar to that found in the Soyuz TMA IDS, it was decided from the start that the final design will include one central main display showing the view from the Soyuz periscope, and two separate screens displaying Orbiter's MFDs. Ideally, input controls for the MFDs would be intuitive, and not require the use of the mouse.

Different methods, including physical buttons, mouse and touch screens were considered. Touch screens were picked as an easy-to use solution that is both more intuitive for the user as compared to mouse controls, and requires little assembly compared to physical buttons or switches (15 buttons per instrument panel would be required). The touch screens each display a single MFD window and can be interacted with by touching different control buttons shown on the displays. Testing with first time users has shown immediate understanding in how to manipulate the instrument panels.

Each display has a resolution of $1024 \times 600$ pixels, with the LCD units measuring about $160 \times 92 \mathrm{~mm}$. The displays have a different aspect ratio (the ratio between width and height of the display or window) to Orbiter's MFDs resulting in unused space around the left and right ends of each screen. This is due to the

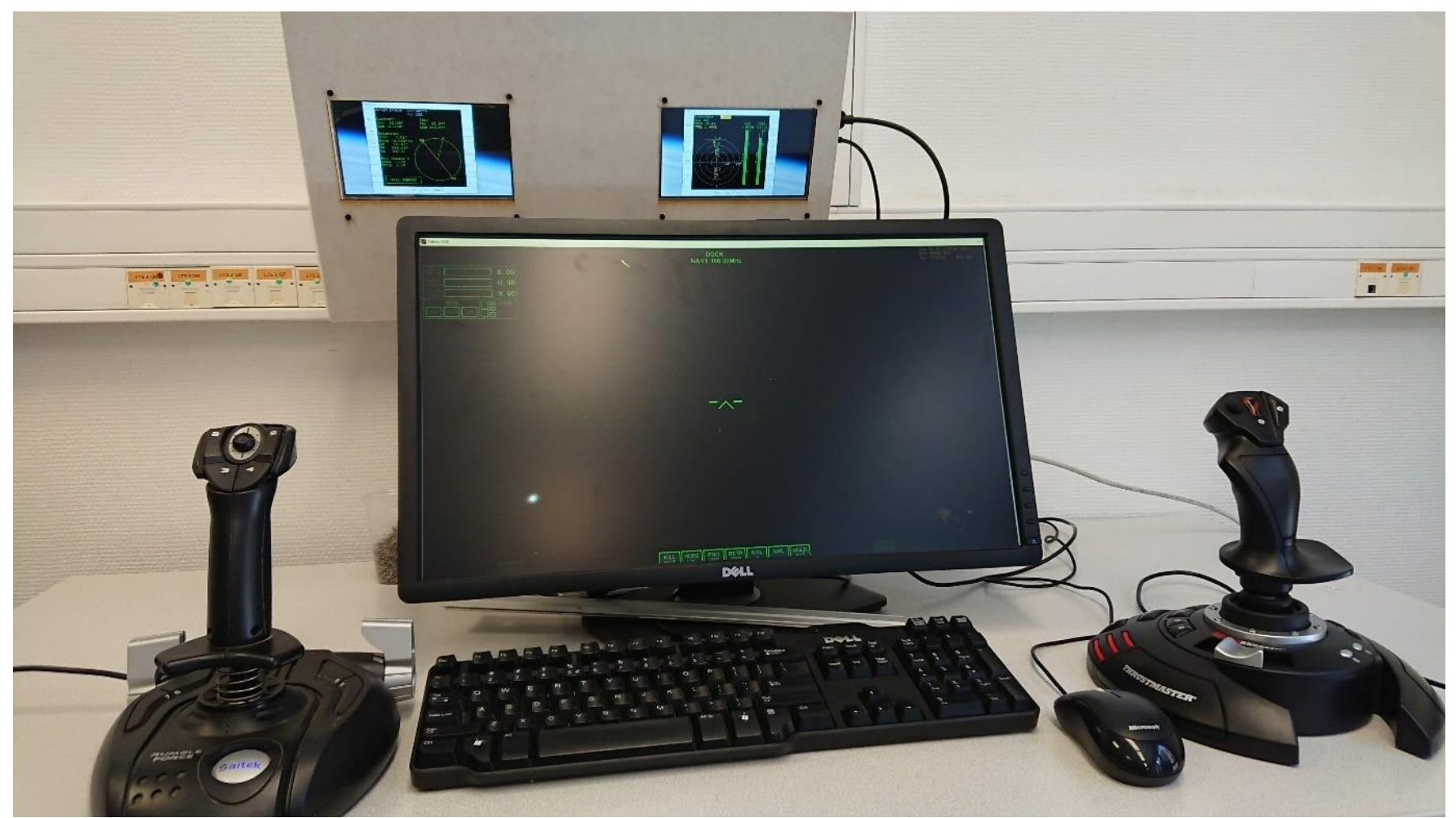

Figure 1: Soyuz Simulator main screen, instrument panels and controls. 


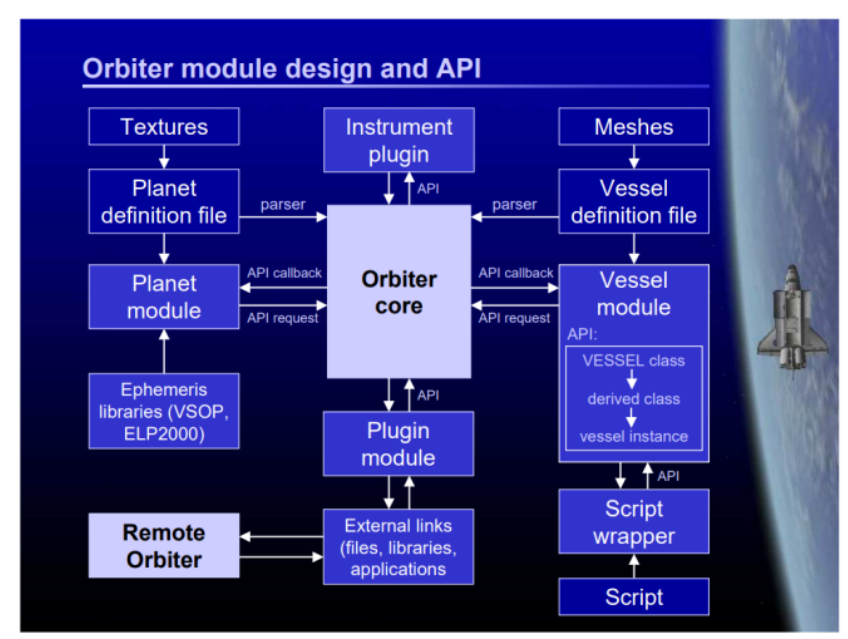

Figure 2: Orbiter Software Architecture [6].

rarity of ready-to-use touch displays with aspect ratios other than 16:9, while Orbiter's MFDs are closer to a 4:3 aspect ratio.

The displays were mounted on a large sheet of plywood that was then affixed to a monitor stand. The monitors connect via HDMI for the video signal, with a separate micro-USB cable needed for touch input and power. This brings the number of connected monitors to 3, requiring the use of 2 ASUS EAH5450 GPUs, as each GPU supports only up to 2 simultaneous video outputs.

By default, all MFDs in Orbiter are rendered in the same window as the rest of the program. Orbiter 2010 comes packaged in with the "ExternalMFD" addon, which when enabled allowing for additional MFDs to be rendered in separate windows. In this design, the ExternalMFD add-on is used to place 2 MFDs on the touch displays. The MFDs are identical in all functionality to those in the main Orbiter window, with all Instruments add-ons automatically supported.

The ExternalMFD windows require to be opened manually during each session, which would be highly inconvenient. This

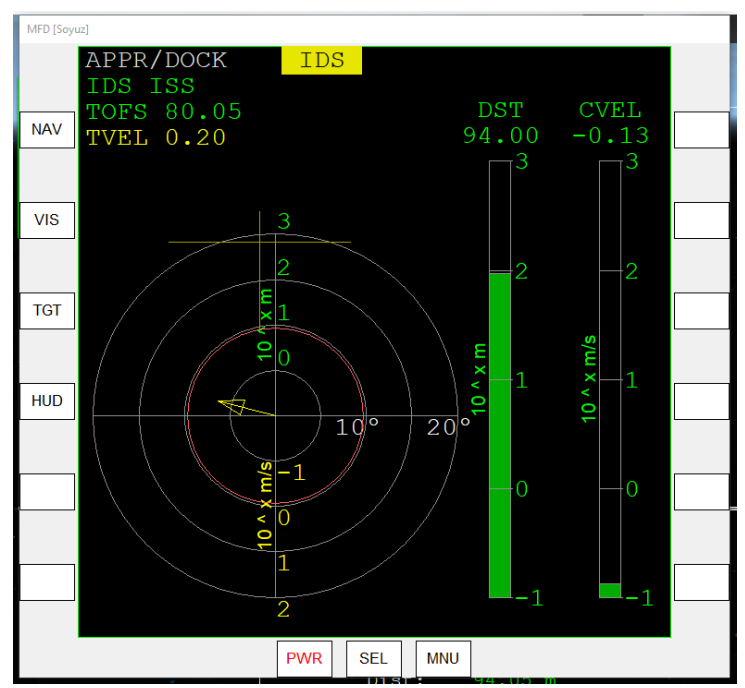

Figure 3: The "Docking" MFD helps keep track of the docking target and align for approach. was automated by using AutoHotkey, a scripting framework for controlling mouse movement, key presses and certain application behaviour in Windows [10]. AutoHotkey provides a complete scripting language, allowing complex preprogrammed and input-based mouse behaviour, and is often used to automatically open and configure graphical applications. In this design, an AutoHotkey script was used to automatically launch Orbiter, open two ExternalMFD windows via scripted key presses and window resizing operations, then drag each window to the appropriate position on the touch displays.

To replicate the Soyuz manual thruster controls, a pair of 3axis analogue USB flight sticks are used. A Thrustmaster T.Flight Stick X and a Saitek Cyborg 3d Rumble Force were used in this case. The sticks are designed for use with flight simulator computer games, with each stick providing 4 analogue (or continuous) input axes, and a number of push buttons. Each stick can be rotated along 2 axes ( $\mathrm{X}$ and $\mathrm{Y}$ ) and twisted along the $\mathrm{Z}$ axis; the 4 th analogue input is provided by a continuous slider on the Thrustmaster stick, and by a rotating axle on the Saitek stick [11] [12].

The 3 axes of each stick are mapped to the manoeuvring thruster groups similarly to the control sticks in the Soyuz spacecraft, with the Thrustmaster placed to the right of the user allowing for attitude control and the Saitek placed to the left allowing for translational control. Control of the main engine is mapped to the slider on the right stick.

While Orbiter is developed with analogue stick input as a possibility, the default version can only accept input from a single device. To add support for multiple analogue devices, the Fly By Wire addon was used [13]. The add-on provides a way to significantly reconfigure all flight stick inputs that are passed to Orbiter, as well as support for multiple analogue input devices.

\section{FINAL VERSION}

The final version of the simulator can let students run through a Soyuz mission from launch to landing or start at the beginning of any major ISS mission stage, such as orbital rendezvous, docking, deorbit and re-entry. The control layout is meant for a single student to use. A short manual was produced for familiarizing ISU students with the simulator controls and principles of spaceflight in Orbiter.

\section{TABLE I. SOFTWARE USED}

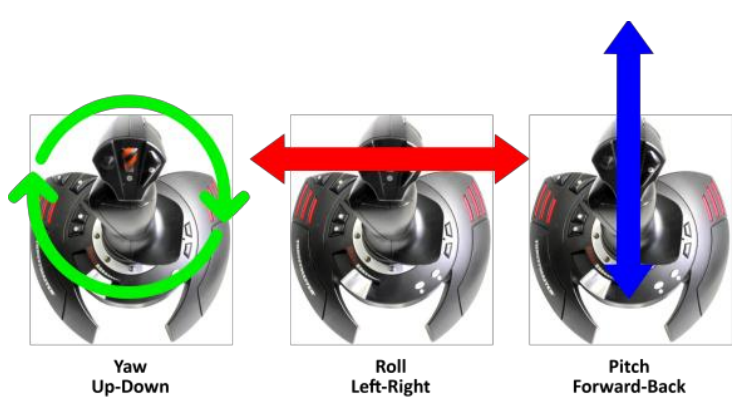

Figure 4: Flight stick control axes, with uses for the right stick (attitude control) then left stick (movement control) respectively. 


\begin{tabular}{|c|c|}
\hline Software & Usage \\
\hline Windows 10 Pro, 64 bit & Operating System \\
\hline AMD Catalyst & Graphics Drivers installation to use 2 \\
& GPUs \\
\hline Orbiter 2010 & Simulator Framework \\
\hline JoyToKey & $\begin{array}{c}\text { Flight Stick input mapping to mouse and } \\
\text { keyboard }\end{array}$ \\
\hline AutoHotkey & Automate opening of ExternalMFD \\
windows
\end{tabular}

TABLE II. SOFTWARE PACKAGES FOR ORBITER

\begin{tabular}{|c|c|}
\hline Package & Features \\
\hline Orbiter 2010 & Orbiter Core \\
\hline Soyuz v1.0 & - Soyuz TMA Spacecraft \\
& - Soyuz Launcher \\
& - Fregat Upper Stage \\
& - Soyuz mission scenarios at different stages in mission \\
& - Can use OrbiterSound if installed \\
\hline ISS v.3.2 & - ISS \\
& - Soyuz TMA Spacecraft (less detailed model, \\
& $\begin{array}{c}\text { overridden by Soyuz v1.0 add-on) } \\
\text { - Progress M1 }\end{array}$ \\
& - Historic Soyuz mission scenarios \\
\hline FlyByWire & - Multiple Flight Stick support and configuration \\
& - Deadzone settings and input axis mapping \\
\hline External & - additional MFDs can be created in separate windows \\
\hline MFD & \\
\hline
\end{tabular}

TABLE III. FULl COMPUTER HARDWARE CONFIGURATION

\begin{tabular}{|c|c|c|c|c|}
\hline Component & Model & $\underset{t}{\operatorname{Amoun}}$ & $\begin{array}{c}\text { Socket/Connectio } \\
\text { n Type }\end{array}$ & $\begin{array}{c}\text { Roug } \\
\text { h } \\
\text { Price } \\
\text { in } \\
\mathbf{2 0 1 9} \\
\text { (eur) }\end{array}$ \\
\hline Processor & $\begin{array}{c}\text { Intel Core } \\
\text { i5-3470 }\end{array}$ & 1 & FCLGA1155 & 80 \\
\hline GPU & $\begin{array}{c}\text { ASUS } \\
\text { EAH5450 }\end{array}$ & 2 & PCI Express & 50 \\
\hline RAM & $\begin{array}{c}\text { DDR3 } \\
\text { Memory }\end{array}$ & $8 \mathrm{~GB}$ & DDR3 DIMM & 30 \\
\hline $\begin{array}{l}\text { Motherboar } \\
\mathrm{d} \text { and other } \\
\text { PC } \\
\text { components }\end{array}$ & N/A & 1 & N/A & 100 \\
\hline $\begin{array}{c}\text { Main } \\
\text { Monitor }\end{array}$ & $\begin{array}{c}\text { Generic } \\
1080 \text { p LCD }\end{array}$ & 1 & DVI & 90 \\
\hline $\begin{array}{c}\text { Touch } \\
\text { Screens }\end{array}$ & $\begin{array}{c}\text { Generic } \\
1024 \text { x } 600 \\
\text { LCD }\end{array}$ & 2 & $\begin{array}{l}\text { HDMI (video) } \\
\text { Micro-USB (touch } \\
\text { input and power) }\end{array}$ & 120 \\
\hline Flight Sticks & $\begin{array}{c}- \\
\text { Thrustmaste } \\
\text { r T.Flight } \\
\text { Stick X } \\
\text { - Saitek } \\
\text { Cyborg 3d } \\
\text { Rumble } \\
\text { Force } \\
\end{array}$ & 2 & USB & 60 \\
\hline $\begin{array}{c}\text { Keyboard } \\
\text { and Mouse }\end{array}$ & & 1 & $2 \times$ USB & 15 \\
\hline
\end{tabular}

\section{Educational Value}

A few trial runs of the simulator were conducted with the help of ISU students that have not interacted with a spaceflight simulator before. Students were given the task of docking the Soyuz to the ISS from a starting position a few hundred metres away. This involved locating a vacant docking port, aligning the Soyuz for approach, approaching the docking port from the correct direction, and docking. An instructor was present to explain how to operate the simulator, give hints on good docking practices and answer the student's questions. First time students took 20 to 50 minutes to complete a full docking.

\section{A video of one of these test runs can be found at:}

\section{https://youtu.be/xeEW5ZFLJh8}

All students on the ISU MSS course have attended a few lectures on the basics of spaceflight mechanics about 6 months prior to when the simulator was tested. While no formal assessment of the simulator's effects on students' understanding of spaceflight mechanics took place, after docking the simulated Soyuz, students have reported feeling immersed while using the simulator, and that their understanding of spacecraft physics has improved.

\section{CONCLUSIONS}

In this paper, the design, capabilities and educational use cases of a low-fidelity Soyuz simulator built at ISU were presented. The simulator is capable of recreating all stages of a typical Soyuz TMA mission. It can be used by students to experiment with orbital mechanics by attempting to rendezvous with the ISS, learn to manoeuvre precisely in micro-gravity, perform docking and control a spacecraft during re-entry. Each of these operations is performed with instruments and controls not too different from those used in the real Soyuz but with a very low learning curve, providing students with an immersive atmosphere that allows them to develop an intuitive understanding of the underlying physics and engineering concepts in spaceflight.

The Orbiter Software's simple controls and standardised MFD instruments help make the simulator approachable for students with only a basic theoretical understanding of the concepts involved. For example, after a few lectures on the basics of orbital mechanics and orbital manoeuvres, a student can try their hand at performing the manoeuvres they learnt about with the simulator, hands-on. Orbiter's scenario system allows for instructors to quickly load pre-made scenarios for each student, while AutoHotkey speeds up the loading of MFDs.

Due to Orbiter's versatility as a spacecraft simulator, and its large user community, a number of improvements can be easily added to the simulator to expand its capabilities both as a teaching tool and for outreach, without additional hardware costs by using community-built add-ons with the presented simulator design. As it is currently set-up, the simulator can be made to simulate spacecraft other than the Soyuz, most notably it can be used to simulate near-future space missions to deep space, helping students understand the distances and manoeuvres needed for interplanetary transfers. Mods that add different hardware support can also be used to extend the simulator with additional hardware to fit more specialised use cases. 


\section{REFERENCES}

[1] A. Siddiqi, Challenge to Apollo, NASA, 2000, p. 249.

[2] S. Siceloff, "Simulators Give Astronauts Glimpse of Future Flights," NASA, 2016. [Online]. Available:

https://www.nasa.gov/feature/simulators-give-astronauts-glimpse-offuture-flights/. [Accessed 4 April 2019].

[3] University of Stuttgart, IRS Institut für Raumfahrtsysteme, "Soyuzsimulator," [Online]. Available: https://www.irs.unistuttgart.de/forschung/Soyuz-Simulator/index.en.html. [Accessed 3 April 2019].

[4] European Space Agency, "Soyuz simulator in Antarctica," 2019. [Online]. Available:

https://www.esa.int/spaceinimages/Images/2019/02/Soyuz simulator in _Antarctica. [Accessed 4 April 2019].

[5] I. Romanov-Chernigovsky and G. Sun, "Building a Low-Fidelity Simulator at ISU. Individual Project Final Report.," International Space University, Strasbourg, 2019.

[6] M. Schweiger, "Orbiter: A Free Spacecraft Simulation Tool," 2004. [Online]. Available: http://download.orbit.m6.net/news/orbiter.pdf. [Accessed 1 April 2019].
[7] O. H. Mods, "Soyuz v1.0," 2016. [Online]. Available: https://www.orbithangar.com/searchid.php?ID=6907. [Accessed 1 April 2019].

[8] O. H. Mods, "International Space Station v.3.2," 2010. [Online]. Available: https://www.orbithangar.com/searchid.php?ID=3737. [Accessed 1 April 2019].

[9] W. Gu and G. Sun, "Low-Fidelity Soyuz Simulator at ISU. Individual Project Final Report.," International Space University, Strasbourg, 2018.

[10] OrbiterWiki, "FlyByWireEGCS - OrbiterWiki," 2009. [Online]. Available: https://www.orbiterwiki.org/wiki/FlyByWireEGCS. [Accessed 30 March 2019].

[11] G. C. S.A., "T.Flight Stick X User Manual," 2015. [Online]. Available: http://ts.thrustmaster.com/download/accessories/pc/joysticks/manuals/tfl ightstickx/tfsx_user_manual.pdf. [Accessed 30 MArch 2019].

[12] Saitek, "Cyborg 3D Rumble Force Stick User Manual," [Online]. Available:

http://www.saitek.com/manuals/Cyborg\%203D\%20Rumble\%20Force\% 20Stick.PDF. [Accessed 30 March 2019].

[13] C. Mallet, "AutoHotkey Documentation," 2014. [Online]. Available: https://www.autohotkey.com/docs/AutoHotkey.htm. [Accessed 3 April 2019]. 